\author{
Commentary on: Mewe R., Gronenschild E. H. B. M., and van den Oord G. H. J., \\ 1985, A\&AS, 62, 197
}

\title{
X-ray emission spectrum of a hot plasma
}

\author{
J. C. Raymond
}

\author{
Center for Astrophysics, 60 Garden St., Cambridge, MA 02138, USA \\ e-mail: jraymond@cfa.harvard.edu
}

In the late 1960s it became apparent that the radiative cooling rate of hot, low-density astrophysical plasmas is dominated by line emission from ions excited by collisions with electrons, along with the bremsstrahlung continuum. Ionization balance calculations were performed for coronal equilibrium, where all the ions are assumed to be in the ground state and collisional ionizations from one ionization state balance recombinations from the next higher ionization state. Cox (1969) presented one of the first cooling models.

By the early 1970s, the need for theoretical X-ray emission spectra of hot plasmas had become more urgent. X-ray observations with proportional counters provided low-resolution $(\Delta E / E$ of a few) spectra of supernova remnants, active stars, accreting compact objects, and the local interstellar background, but it was not possible to invert the measured pulse height distribution to obtain the spectrum. Instead, a set of theoretical models would be folded through the instrument response and compared with the measured pulse heights to find the best fit. Tucker (1971), Landini (1970), and Mewe (1972a) provided the first such models. The atomic rates available were sparse, and there were large disagreements between different estimates of many important rates. Interpolation, extrapolation, and crude Gaunt factor approximations were required. Choosing among discrepant theoretical estimates of the rate coefficients was an art. Mewe (1972b) in particular provided interpolation formulae for several important isoelectronic sequences.

In the middle 1970s, X-ray emission calculations that made use of the increasing amount of atomic data appeared (Kato 1976; Raymond 1977; Stern 1978; Gaetz 1983). Theoretical calculations of the relevant atomic rates became more reliable with the advent of bigger computers and large codes using the distorted wave or close coupling methods. It remained difficult to choose among different computed cross sections, because the computer power available at that time was seldom adequate for including a very good model of the target ion's wave function, but laboratory benchmarks for a few transitions helped to sort out the uncertainties. The spectrum models were widely used with data from satellites to obtain the temperatures, luminosities, and elemental abundances of astrophysical plasmas ranging from the stellar corona to supernova remnants. Parameters were obtained by $\chi^{2}$ minimization under the assumption that the spectrum model was free of error.

During this time, Rolf Mewe and his collaborators continued to extend the Mewe (1972a) calculation in several direc- tions. Mewe (1975) extended his model to longer wavelengths. Mewe (1978) provided formulae for the contribution of recombination to excited levels to the emission lines of He-like ions, and Mewe (1977) gave interpolation formulae for oscillator strengths. Gronenschild (1978) improved the treatment of continuum emission. The paper by Mewe (1985) is to some extent the culmination of this series of papers by Rolf Mewe and collaborators, and it gives a state-of-the-art model of X-ray emission from hot plasma in collisional ionization equilibrium. However, it also provides the basis for the continuing development of the model. The current version is known as Mekal, after Rolf Mewe and Jelle Kaastra added Fe emission lines computed by Duane Liedahl with HULLAC, and this updating is essential to the model's continuing usefulness.

The 1980s brought higher resolution X-ray spectroscopy to astrophysics with the SSS and FPCS instruments on the Einstein satellite, and that meant it became possible to derive temperature distributions and densities along with more reliable elemental abundances. It also meant that more accurate theoretical $\mathrm{X}$-ray emission models, with more emission lines and higher accuracy, were required. Most of the models used for fitting X-ray data used pre-computed tables of the ionization balance in collisional equilibrium, and they were not appropriate for the timedependent ionization in supernova remnants and photoionized plasma in X-ray binaries. A separate set of models emerged to handle those cases (Itoh 1978; Hamilton 1983; Kallman 1982), and Mewe's code was extended to time-dependent ionization calculations for solar flares and supernova remnants (Mewe 1980; Sylwester 1980; Gronenschild 1982).

Since that time, the amount of atomic data has mushroomed, as have computer speed and memory. Atomic stucture and collision codes, such as HULLAC, FAC, and RMATRIX, produce enormous amounts of atomic data that is more accurate than the older data. Modern codes, such as APEC/APED (Smith 2001) and CHIANTI (Landi 2006), solve the population matrices for hundreds of energy levels and predict intensities of many thousands of emission lines. Processes such as recombination to excited levels, which are most important for the $\mathrm{H}$ like and He-like isoelectronic sequences, are beginning to be included for other ions as well.

When spectral resolution improved with the grating instruments on Chandra and XMM, a debate arose about the relative usefulness of global fitting to a model such as Mekal, as opposed to measuring individual line ratios sensitive to density, tempera- 
ture, or abundance. The line ratio method, which has dominated in solar physics for many years, has the advantage of giving welldefined numbers, such as the density averaged over the region that produces the O VII lines, and it also has the advantage that one can restrict attention to a few bright lines for which relatively accurate atomic rates are available. The disadvantages are that one is throwing away the information contained in all the weaker lines and that high-resolution spectra are only available for the brightest sources.

The Mewe (1985) model, along with its companion paper on continuum processes (Mewe 1986), includes all the most important atomic processes for X-ray emission from a hot, low, or moderate density plasma, and it is marked by careful evaluation of the atomic rates for individual spectral lines. It gives a massive table of fits to excitation rates for individual spectral lines, so it has been very useful to spectroscopists, but it has mostly been used as a black box inside spectral fitting procedures. Over the years it has been used to analyze a huge number of X-ray spectra. Mewe and his collaborators tended to be most interested in stellar coronae, and they used XMM, Chandra, and EUVE to investigate the emission measure distributions and elemental abundances of a wide range of stars.

As pointed out by Nancy Brickhouse, one of the most important aspects of the Mekal model is that it, along with a similar model by Raymond (1977), provided standard descriptions of the emission from thermal plasmas, so that analyses by different groups could be intercompared without the worry that different temperature or abundance results simply stemmed from the use of different sets of X-ray emission models. These particular models became standard partly because they were convenient, and partly because they were included in the standard fitting packages, such as XSPEC (Arnaud 1996).

As the quality of high-resolution X-ray spectra improves, discrepancies with the models continue to arise. Additional spectral lines must be added and existing atomic rates must be improved. For the coronal equilibrium plasma considered by
Mewe (1985), the improvements in ionization and recombination rate coefficients (Bryans 2006,2009) are especially important, but comparison with solar spectra (Bryans 2009) shows that serious problems still remain for some isoelectronic sequences. Further development of X-ray spectrum models is needed as theoretical calculations and laboratory measurements improve the atomic rates, both for exploiting the existing XMM and Chandra grating spectra and preparing for spectra from the NeXT and IXO missions.

\section{References}

Arnaud, K. A., in Astronomical Data Analysis Software and Systems V, ASP Conf. Ser. 101, ed. G. H. Jacoby, \& J. Barnes (San Francisco: A.S.P.), 17 Bryans, P., Badnell, N. R., Gorczyca, T. W., et al. 2006, ApJS, 167, 343 Bryans, P., Landi, E., \& Savin, D. W. 2009, ApJ, 691, 1540 Cox, D. P., \& Tucker, W. H. 1969, ApJ, 157, 1157 ct69

Gaetz, T. J., \& Salpeter, E. E. 1983, ApJS, 52, 155

Gronenschild, E. H. B. M., \& Mewe, R. 1978, A\&AS, 32, 283

Gronenschild, E. H. B. M., \& Mewe, R. 1982, A\&AS, 48, 305

Hamilton, A. J. S., Chevalier, R. A., \& Sarazin, C. L. 1983, ApJS, 51, 115 Itoh, H. 1978, PASJ, 29, 813

Kallman, T. R., \& McCray, R. 1982, ApJS, 50, 263

Kato, T. 1976, ApJS, 30, 397

Landi, E., Del Zanna, G., Young, P. R., et al. 2006, ApJS, 162, 261

Landini, M., \& Monsigniori Fossi, B. C. 1970, A\&A, 6, 468

Mewe, R. 1972a, Sol. Phys., 22, 459

Mewe, R. 1972b, A\&A, 20, 215

Mewe, R. 1975, Sol. Phys., 44, 383

Mewe, R. 1977, A\&A, 59, 275

Mewe, R., \& Schrijver, J. 1978, A\&A, 65, 99

Mewe, R., \& Gronenschild, E. H. B. M. 1981, A\&AS, 45, 11

Mewe, R., Schrijver, J., \& Sylwester, J. 1980, A\&AS, 40, 323

Mewe, R., Gronenschild, E. H. B. M., \& van den Oord, G. H. J. 1985, A\&AS, 62, 197

Mewe, R., Lemen, J. R., \& van den Oord, G. H. J. 1986, A\&AS, 65, 511

Raymond, J. C., \& Smith, B. W. 1977, ApJS, 35, 419

Smith, R. K., Brickhouse, N. S., Liedalh, D. A., \& Raymond, J. C. 2001, ApJ, 556, L91

Stern, R., Wang, E., \& Bowyer, S. 1978, ApJS, 37, 195

Sylwester, J., Mewe, R., \& Schrijver, J. 1980, A\&AS, 40, 335

Tucker, W. H., \& Koren, M. 1971, ApJ, 168, 283 